\title{
CMV Disease of Upper GI Tract in Immunocompetent Host: Two Case Reports and Brief Review of Literature
}

\author{
Nikhil Mahajan ${ }^{1,2}$ \\ Balwinder Singh ${ }^{1}$ \\ ${ }^{1}$ Department of Gastroenterology, Shrimann Hospital, Jalandhar, \\ Punjab, India \\ ${ }^{2}$ Department of Gastroenterology, Medanta-The Medicity, \\ Gurugram, Haryana, India \\ ${ }^{3}$ Department of Pathology, AFCME, New Delhi, India \\ ${ }^{4}$ Department of Gastro-Pathology, Medanta-The Medicity, Gurugram, \\ Haryana, India \\ ${ }^{5}$ Department of Pathology, ASCOMS, Jammu, J \& K, India \\ ${ }^{6}$ Department of Pathology, Shrimann Hospital, Jalandhar, Punjab, \\ India
}

J Digest Endosc 2021;12:249-252.
Lipika Lipi $^{4}$ Rini Sharma ${ }^{5}$ Navleen Arora ${ }^{6}$

Address for correspondence Nikhil Mahajan, MBBS, MD (med), DNB (Gastroenterol), 261, Nai Basti, Jammu Cantt, Jammu, J and K., Pin 180003, India (e-mail: niks.mahajan54@gmail.com).

\begin{abstract}
Keywords

- cytomegalovirus (CMV)

- esophageal ulcer

- gastric ulcer

- immunocompetent

- upper gastrointestinal tract

Gastrointestinal cytomegalovirus (GI-CMV) infection is well-reported in immunocompromised individuals and those on chemotherapy or other immunosuppressive medications. Rarely, CMV infection of gastrointestinal tract (GIT) occurs in immunocompetent individuals. Colon is the most common site of $\mathrm{Gl}$ involvement with CMV. However, in immunocompetent individuals, upper GIT involvement is more common. Clinical spectrum of GI-CMV disease is variable and depends on the location and severity of the disease. Prompt diagnosis and treatment aids in reducing morbidity and mortality related to CMV involvement in GI tract. We present two interesting cases of CMV-induced Gl ulcers in immunocompetent individuals with brief review of diagnostic and treatment challenges associated with CMV disease in immunocompetent individuals.
\end{abstract}

\section{Introduction}

Cytomegalovirus (CMV) is a common human viral infection, with high seroprevalence, affecting 40 to 100 percent of adults. $^{1}$ Although immunocompetent patients may be infected with CMV, it rarely causes active disease in them. ${ }^{2,3}$ In healthy immunocompetent individuals, majority of primary CMV infections are either asymptomatic or present as a mild mononucleosis-like syndrome. ${ }^{4}$ Overall, colon is the most common site of involvement followed by esophagus. But in immunocompetent individuals, upper gastrointestinal tract (GIT) involvement is more common. ${ }^{3}$ Symptomatic patients with CMV esophagitis or gastritis may present with fever, weight loss, dysphagia/odynophagia, nausea/vomiting, abdominal pain or, rarely, GI bleed or stricture formation. There is no pathognomonic endoscopic feature of CMV disease. ${ }^{5}$ Definitive diagnosis depends heavily on biopsy findings. Finding cytomegalic cells with CMV inclusion bodies is critical to the diagnosis. ${ }^{1}$ Unlike immunocompromised patients, not all immunocompetent patients require specific antiviral treatment for CMV.
DOI https://doi.org/ 10.1055/s-0040-1716449. ISSN 0976-5042.
(C) 2022. Society of Gastrointestinal Endoscopy of India. All rights reserved.

This is an open access article published by Thieme under the terms of the Creative Commons Attribution-NonDerivative-NonCommercial-License, permitting copying and reproduction so long as the original work is given appropriate credit. Contents may not be used for commercial purposes, or adapted, remixed, transformed or built upon. (https://creativecommons.org/ licenses/by-nc-nd/4.0/)

Thieme Medical and Scientific Publishers Pvt. Ltd., A-12, 2nd Floor, Sector 2, Noida-201301 UP, India 

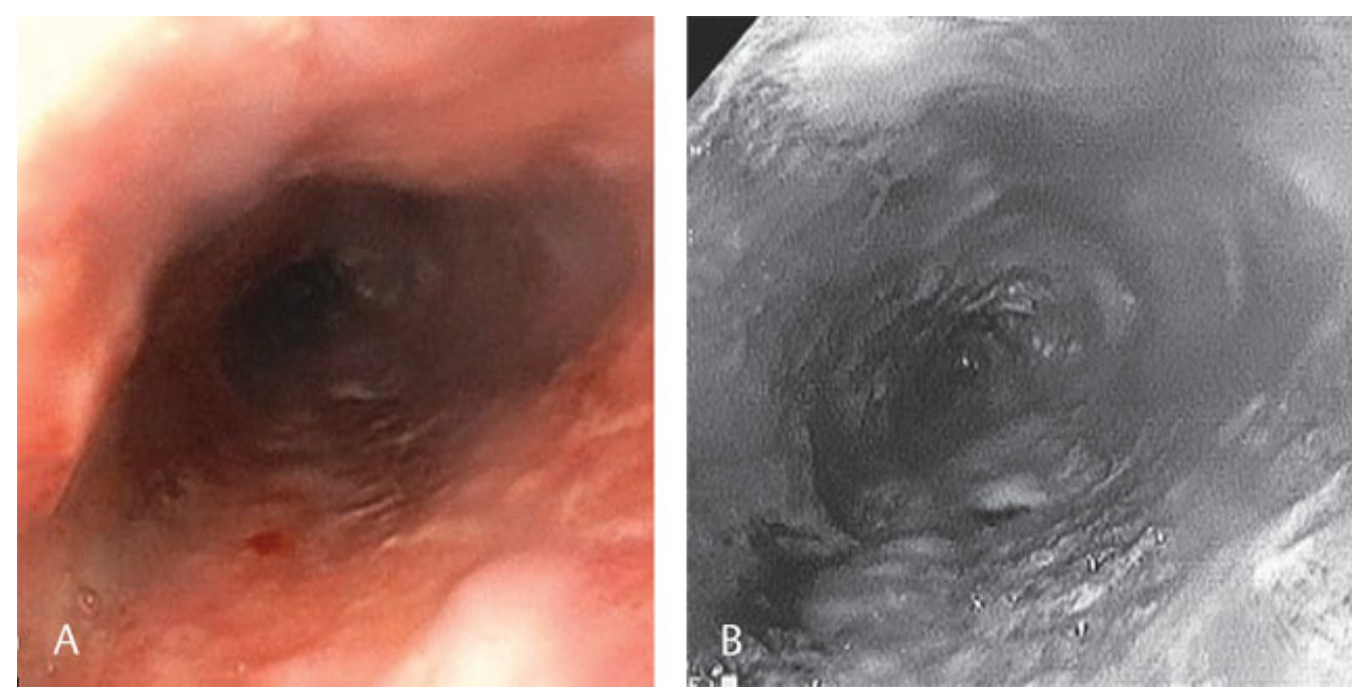

Fig. 1 Mid-esophagus: (A) white light image (WLI) showing patches of normal mucosa amidst diffuse ulceration; (B) narrow band image (NBI) showing normal mucosal patches in light blue color highlighted by arrow and ulcerated mucosa in reddish brown color.

Because CMV esophagitis/gastritis has rarely been described in immunocompetent hosts, our cases add up to the current literature of GI-CMV involvement in immunocompetent hosts.

\section{Case Reports}

\section{Case 1}

A 71-year-old frail looking male with no known comorbidities was admitted with history of fever and generalized weakness. During hospital stay, patient complained of odynophagia. Upper gastrointestinal endoscopy (UGIE) showed a circumferential shallow ulcer starting at $35 \mathrm{~cm}$ from

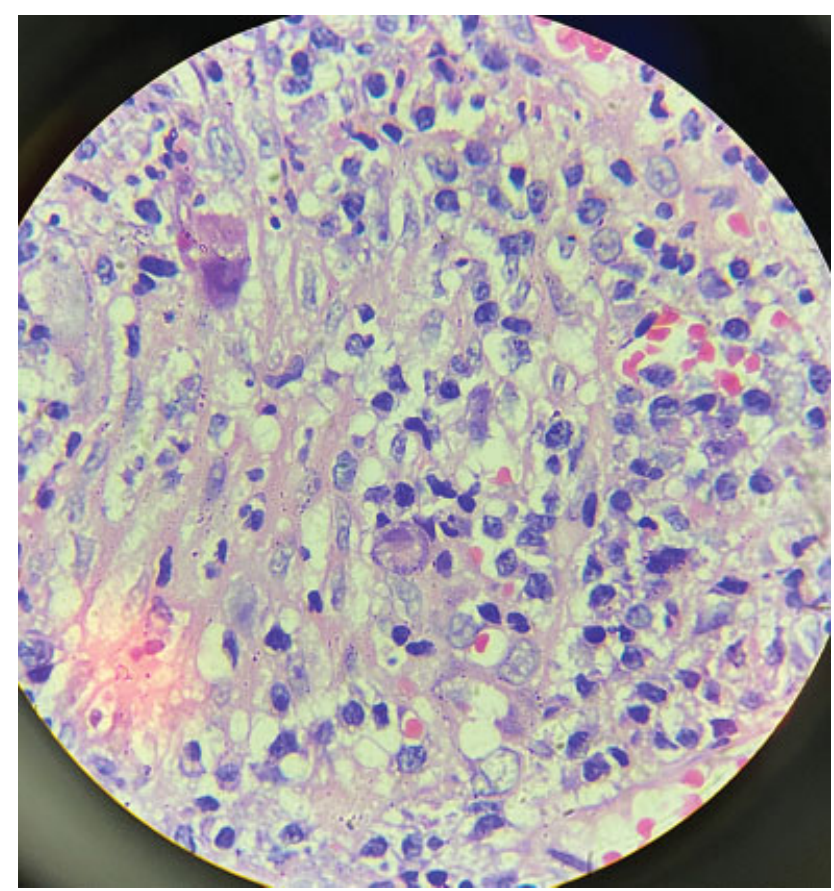

Fig. 2 Dense lymphomononuclear infiltrate with fibroblasts showing cytomegaly with intranuclear inclusions. $\mathrm{H} \& \mathrm{E} \times 400 \mathrm{x}$. incisors till GE junction at $38 \mathrm{~cm}$ (-Fig. 1). Biopsies were obtained from the ulcer edges as well as from the base, which on histopathology showed ulcerated esophageal mucosa with dense lymphomononuclear infiltrate with few endothelial cells and fibroblasts showing marked enlargement and large basophilic intranuclear inclusions surrounded by clear halos typical of CMV inclusions (-Figs. 2-3). Immunohistochemical staining for CMV was not done in this case, as hematoxylin and eosin stain showed inclusion bodies, and patient was not willing to spend more money for immunohistochemistry (IHC) testing. He was started on antiviral therapy. Intravenous gancyclovir was given for initial 5 days followed by oral valgancyclovir for total of 3 weeks, both in induction doses, along with other supportive treatment. His symptoms improved with the given treatment. Repeat UGIE after 3 weeks of induction therapy showed evidence of healed ulcers. Valgancyclovir was continued in maintenance dose for a total of 8 weeks treatment.

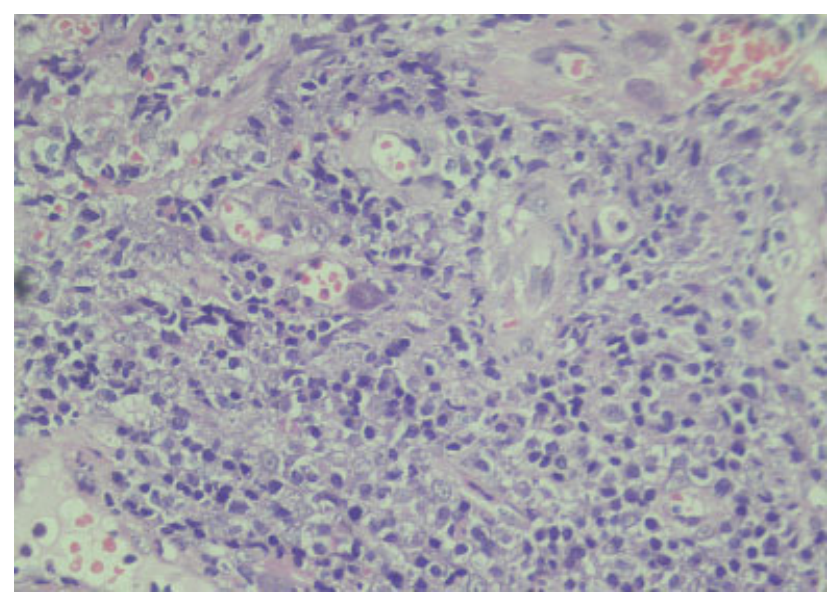

Fig. 3 Endothelial cells showing cytomegaly with intranuclear inclusions. $H \& E \times 200 x$. 


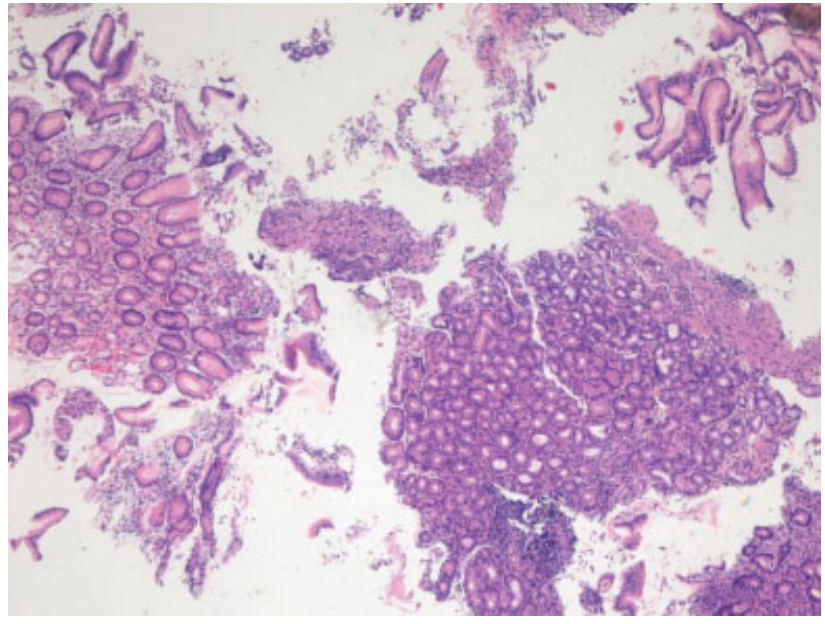

Fig. 4 Gastric mucosa showing focal ulceration and moderate mixed inflammatory infiltrate. $\mathrm{H} \& \mathrm{E} \times 40 \mathrm{x}$.

\section{Case 2}

A 55-year-old normotensive, nondiabetic healthy female was admitted with epigastric pain abdomen, nausea, vomiting and low-grade fever. UGIE showed multiple subcentimetric nodular lesions with surrounding erythema on greater curvature of stomach. Biopsies were taken. Histopathology findings were similar to the first case. Section showed gastric mucosa with ulceration and moderate active as well as chronic inflammation. Few endothelial cells as well as fibroblasts showed cytomegaly with intranuclear inclusions (-Fig. 4). These inclusions were immunoreactive to antibodies against CMV antigen on IHC (-Fig. 5). She was managed with proton pump inhibitors and other supportive treatment. Specific antivirals were not given. Her symptoms settled within 3 to 5 days. Repeat UGIE showed no ulcers.

These case reports show the rare possibility of esophageal and gastric CMV disease even in immunocompetent host. Decision of treatment with antivirals should be individualized after weighing benefits over potential toxicity.

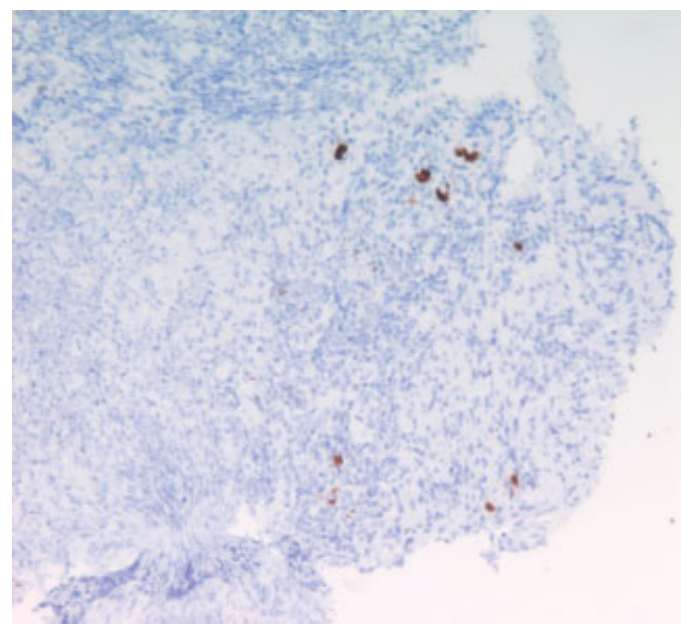

Fig. 5 The inclusions are immunoreactive to antibody against cytomegalovirus (CMV) antigen. IHC $\times 100 x$.

\section{Discussion}

CMV is a double-stranded DNA virus belonging to the Herpesviridae family. CMV disease is rare in immunocompetent individuals, as cell-mediated host immune response prevents the development of overt CMV disease. ${ }^{3}$ The diagnosis of GICMV disease in immunocompetent individuals is difficult because of very low incidence/prevalence, broad tissue tropism of CMV and nonspecific presenting symptoms. However, in the recent past, CMV disease even in immunocompetent individuals has been increasing with more involvement of the upper gastrointestinal tract (GIT). Critically ill or elderly patients, because of their suppressed immunity, are at increased risk of CMV disease. ${ }^{6}$ In a recent retrospective study by Chaemsupaphan et al, $173 \mathrm{GI}-\mathrm{CMV}$ patients were evaluated. ${ }^{7}$ Of these, 56 were immunocompetent and 117 immunocompromised. Immunocompetent patients were significantly older than immunocompromised patients ( 73 vs. 48.6 years, $p<0.0001$ ), with slight male predominance in all GI-CMV cases. However, they had also included individuals with diabetes, chronic kidney disease and chronic liver disease in the immunocompetent group. This might lead to a biased assessment of the immune status of patients. Out of our two patients, one was an elderly male and the other was middle-aged female and both had upper GI involvement similar to what has been described in the most available literature. However, Chaemsupaphan et al in their retrospective analysis found lower GI involvement more in both immunocompetent and immunocompromised patients. This difference from other stated literature could be because of inclusion of comorbidities like chronic liver disease (CLD) and chronic kidney disease (CKD) in the immunocompetent group. Chaemsupaphan et al noted GI bleed as the leading presentation in immunocompetent patients, whereas diarrhea and abdominal pain were more common in immunocompromised patients. Endoscopic findings are nonspecific, with mucosal ulceration being the most common. ${ }^{8}$ Both our patients had painful mucosal lesions in the upper GI tract. Despite this, morbidity and mortality seem to be lower than in immunocompromised patients. Successful diagnosis depends upon diligent study of histopathology slides to look for inclusion bodies. ${ }^{1}$ IHC aids in diagnosis if inclusion bodies are difficult to find in hematoxylin and eosin stain but is not sine-qua-non for making a diagnosis. Serum antibodies, in situ hybridization and PCR for CMV DNA may be the possible additions but generally are not useful. ${ }^{9}$ Blood PCR indicates presence of CMV and not definitely CMV disease, although quantitative blood PCR can help in differentiating asymptomatic carriage from active disease. Available data highlights the importance of blood CMV PCR in immunocompromised patients compared with immunocompetent ones. In retrospective data, ${ }^{7}$ only 60 percent of immunocompetent GICMV disease patients showed viral load in blood PCR compared with 87 percent in immunocompromised patients. During antiviral treatment, repeat blood PCR levels can be helpful. Decreasing or undetected PCR levels compared with baseline indicate good response to therapy and could help in optimizing the duration of therapy needed, although this needs to be validated with more studies. Even symptomatic CMV infections in immunocompetent individuals usually require supportive 
care only. ${ }^{10}$ Current limited evidence suggests that antiviral therapy is appropriate for immunocompetent patients with clinical risk factors such as severe CMV disease or advanced age. Both our patients were immunocompetent. Inclusion bodies could be detected in both the cases under hematoxylin and eosin stain. In one patient, IHC was applied to confirm the diagnosis, in view of its availability and no financial constraints with regard to the patient. Blood PCR was not done for either patient, as diagnosis was already confirmed on histopathology. Our first case was a 71-year-old frail male patient with odynophagia who was not able to take anything by mouth. In this patient, keeping in view his old age, intolerant pain and immune senescence, we started antivirals. Maintenance therapy was also administered to him. Total duration of therapy given was 8 weeks. Most studies mention 3 to 4 weeks of antiviral therapy is sufficient for immunocompetent patients, with selective use of prolonged maintenance course of antiviral for patients with CMV involvement of other organs like CMV retinitis or ongoing immunosuppression to reduce the risk of recurrence. ${ }^{11,12}$ Our patient was old and frail with severe odynophagia at baseline, so maintenance course of antiviral was given to achieve complete remission and reduce the risk of recurrence. Our second patient was a middle-aged, well-built female with no evidence of immunosuppression/depletion, so antivirals were not given. She responded well to the given supportive care.

Our cases highlight the paramount importance of early diagnosis and targeted treatment of upper GI-CMV disease, which implies a high index of clinical suspicion, even in immunocompetent patients. Low threshold for endoscopic examination is crucial to early diagnosis. Advanced age is a risk factor for CMV disease in immunocompetent patients, which is in line with immune senescence. ${ }^{13}$ In the immunocompetent patients, clinical judgment should be used to determine which patients should undergo antiviral therapy after weighing the benefits against potential toxicity.

Conflicts of Interest

None declared.

\section{References}

1 Bernard S, Germi R, Lupo Jet al.Symptomatic cytomegalovirus gastrointestinal infection with positive quantitative real-time PCR findings in apparently immunocompetent patients: a case series. Clin Microbiol Infect 2015;21(12):1121.e1-1121.e7

2 Altman C, Bedossa P, Dussaix E, Buffet C. Cytomegalovirus infection of esophagus in immunocompetent adult. Dig Dis Sci 1995;40 (03):606-608

3 Hwang JB, Park MH, Lee BYet al.Clinical quiz. Cytomegalovirus infection. J Pediatr Gastroenterol Nutr 2006;42(05):607-608

4 Horwitz CA, Henle W, Henle Get al.Clinical and laboratory evaluation of cytomegalovirus-induced mononucleosis in previously healthy individuals. Report of 82 cases. Medicine (Baltimore) 1986;65(02):124-134

5 Wilcox CM, Diehl DL, Cello JP, Margaretten W, Jacobson MA. Cytomegalovirus esophagitis in patients with AIDS. A clinical, endoscopic, and pathologic correlation. Ann Intern Med 1990;113 (08):589-593

6 Al-Omari A, Aljamaan F, Alhazzani W, Salih S, Arabi Y. Cytomegalovirus infection in immunocompetent critically ill adults: literature review. Ann Intensive Care 2016;6(01):110

7 Chaemsupaphan T, Limsrivilai J, Thongdee Cet al.Patient characteristics, clinical manifestations, prognosis, and factors associated with gastrointestinal cytomegalovirus infection in immunocompetent patients. BMC Gastroenterol 2020;20(01):22

8 Iwamuro M, Kondo E, Tanaka Tet al.Endoscopic manifestations and clinical characteristics of cytomegalovirus infection in the upper gastrointestinal tract. Acta Med Okayama 2017;71(02): 97-104

9 Himoto T, Goda F, Okuyama Het al.Cytomegalovirus-associated acute gastric mucosal lesion in an immunocompetent host. Intern Med 2009;48(17):1521-1524

10 Goff JS. Infectious causes of esophagitis. Annu Rev Med 1988; 39:163-169

11 Tan BH. Cytomegalovirus Treatment. Curr Treat Options Infect Dis 2014;6(03):256-270

12 Marques S, Carmo J, Pinto D, Bispo M, Ramos S, Chagas C. Cytomegalovirus disease of the upper gastrointestinal tract: a 10-year retrospective study. GE Port J Gastroenterol 2017;24(06): 262-268

13 Maiorana A, Baccarini P, Foroni M, Bellini N, Giusti F. Human cytomegalovirus infection of the gastrointestinal tract in apparently immunocompetent patients. Hum Pathol 2003;34(12): $1331-1336$ 